\title{
MAC Performance Modeling of IEEE 802.15.6 -based WBANs over Rician-faded channels
}

\author{
Saeed Rashwand \\ Department of Computer Science \\ University of Manitoba
}

\author{
Jelena Mišić \\ Department of Computer Science \\ Ryerson University
}

\author{
Vojislav Mišić \\ Department of Computer Science \\ Ryerson University
}

\begin{abstract}
Since the signal transmission in WBANs takes place around or in the human body, the channel fading significantly affects the error performance of the networks. In this paper, we investigate MAC performance of an IEEE 802.15.6-based WBAN operating over a Rician-faded channel. We deploy Bit Error Rate (BER) as a function of channel quality, diversity order, and Signal to Noise Ratio (SNR) values for all User Priorities (UPs) in analytical and simulation models to evaluate the MAC performance. We study how varying Signal to Noise Ratio (SNR) of all UPs affects the MAC performance. Our results indicate that data frame sizes and channel quality between a node and the hub are the most effective parameters on PHY/MAC performance of a WBAN.
\end{abstract}

\section{INTRODUCTION}

The IEEE 802.15.6 standard as the communication standard for Wireless Body Area Networks (WBANs) has been optimized for low power devices and operation on, in or around the human body [1]. Since the signal transmission in WBANs takes place around or in the human body, the channel fading significantly affects the error performance of the networks. In this paper, we investigate the impacts of the Bit Error Rate (BER), which is caused by the channel fading, on MAC level performance of IEEE 802.15.6 CSMA-based WBANs. We evaluate MAC performance descriptors of the networks based on the Signal to Noise Ratio (SNR) values for different UPs. We assume Rician-faded channels between the nodes and the hub in the network with unequal Rician factors. We also assume that the WBAN nodes deploy the QPSK modulation scheme to achieve the highest data frame transmission rate in ISM bands. The BER values for different nodes are calculated according to the channel quality, diversity level, and SNR values. The obtained BER values for different UPs are used for calculating the data frame error rates of the UPs in the network. Thereafter, we calculate the data frame response times for all UPs are computed.

In addition, because the channel fading in WBANs is quite strong and significantly affects the quality of the received signal, diversity is an important mechanism to improve the error performance of the networks [2]. In this paper, we also investigate the effects of the diversity level on MAC performance measures of WBANs.

To our best knowledge, there is not currently any work in the literature to investigate the effects of fading channels on MAC performance of the IEEE 802.15.6-based WBANs. The remainder of this paper is organized as follows: Section II addresses the related work. In Section III the analytical model is described. Section IV presents the MAC performance of an IEEE 802.15.6-based WBAN over Rician-faded channels through several experiments. Finally, Section V concludes the paper summarizing the findings of the study.

\section{Channel Modeling For WBANs}

In this section, we review the research studies on the channel modeling for WBANs. According to the recent studies, the propagating wave is mostly diffracted around the human body rather than passing through it. Hence, the path loss in WBANs is very high specially when the transmitter and the receiver are shadowed by a part of the body [3]. In the literature, there is a limited research to model the fading channels for WBANs.

In [3], [4] it is shown that Lognormal and Nakagami-m distributions are appropriate for modeling the WBAN fading channels, respectively. However, it is more accepted that Rician distribution is the best suited scheme for modeling the small-scale fading of WBAN channels [5], [6], [7]. There are other studies indicating that Rician distribution is the best option for WBAN channel modeling [8], [9]. We employ the results in [5] for the fading channel modelling.

To analyse the error performance of a wireless system, for each combination of communication type (modulation/detection) and the channel fading the average BER of the system is calculated. The statistical calculation of BER at the physical layer is utilized to compute the Packet Error Rate (PER) at the higher layers.

There is a large body of work in the literature which models the error performance of wireless channels. However, a few studies consider QPSK as the modulation scheme of the wireless systems. The authors in [10], [11] developed an expression for computing BER of DQPSK over a slow Rician fading channel. By deploying the alternate representations of classic functions, such as Gaussian and Marcum Q-functions, the authors in [12] developed a unified framework for evaluating the error-probability performance of coherent, differentially coherent, and noncoherent communications over generalized fading channels. The average BER is analysed for M-PSK modulation over Rician fading channels considering the linear diversity in [13]. The authors in [14] derived closed-form expressions for calculating the average BER for a class of modulation schemes over Rician fading channels. 
The authors in [15] computed BER of linearly modulated signals, including DBPSK, DQPSK, and D8PSK, in Rician, Rayleigh, and Nakagami-m fading channels for coherent receivers with $L$-th order MRC (Maximal Ratio Combining) diversity. We use the expression for BER based on SNR derived in [15] to investigate the error performance of the WBAN channels. The BER of QPSK in Rician fading channels is given as follows [15]:

$$
B E R_{Q P S K-\text { Rice }}=G\left(0, \frac{\pi}{2}, \overline{\gamma_{b}}, L, K_{R}, 1\right)
$$

where $\overline{\gamma_{b}}=\frac{\overline{\gamma_{c}}}{2}, \overline{\gamma_{c}}=\frac{1}{2 N_{0}}$ represents the average SNR per channel, $N_{0}$ is the power spectral density of the complex Gaussian random processes for the channels, and $K_{R}$ is the Rician factor, and we have

$$
\begin{aligned}
& G\left(\theta_{1}, \theta_{2}, \bar{\gamma}, L, K_{R}, d\right)= \\
& \frac{e^{-L K_{R}}}{\pi} \int_{\theta_{1}}^{\theta_{2}} \frac{\exp \left(\frac{L K_{R}}{1+\left(\bar{\gamma} d^{2} /\left(K_{R}+1\right) \sin ^{2} \theta\right)}\right)}{\left[1+\left(\bar{\gamma} d^{2} /\left(K_{R}+1\right) \sin ^{2} \theta\right)\right]^{L}} d \theta
\end{aligned}
$$

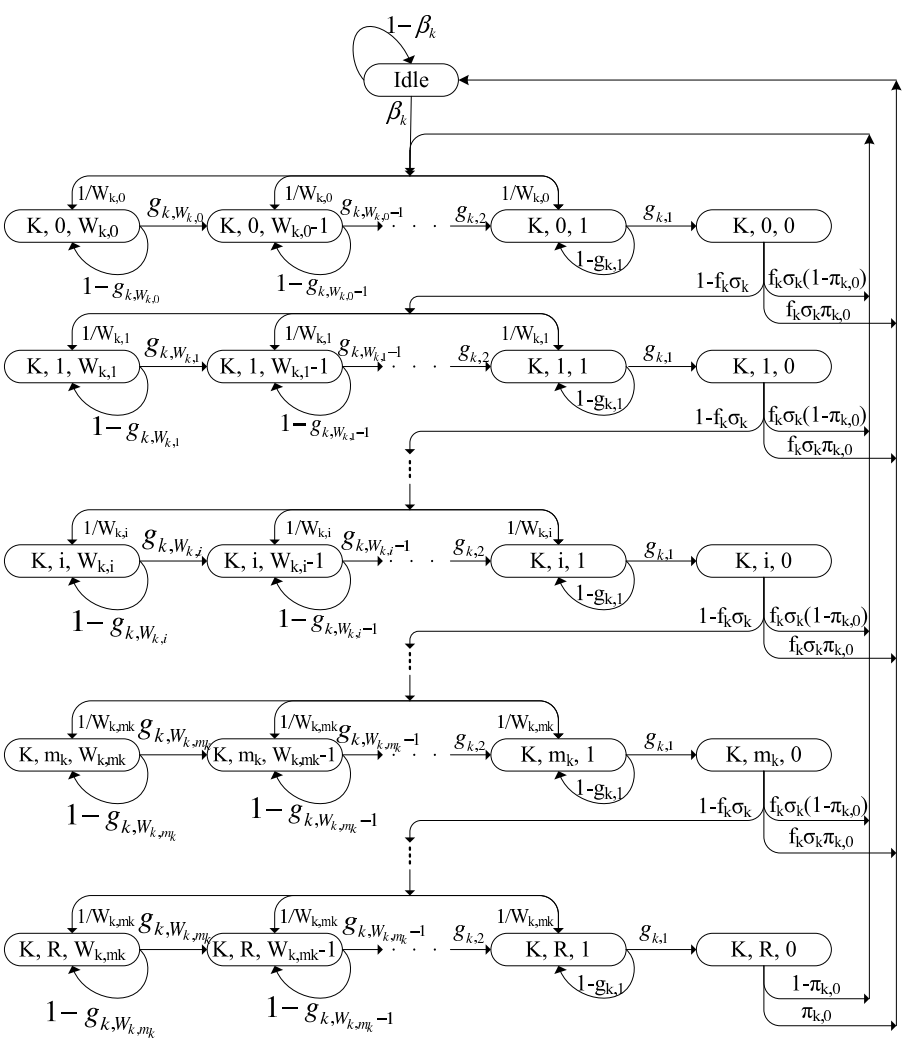

Fig. 1. Developed 3-dimensional DTMC for $\mathrm{UP}_{k}$

\section{Analytical Model}

In this section, we extend the developed analytical model in [16] to investigate the error performance of an IEEE 802.15.6 CSMA-based WBAN. Due to lack of space we only present the model components which are different than [16]. The deployed parameters in the model are defined in Table I. We

\begin{tabular}{|c|c|}
\hline Parameter & Description \\
\hline$k$ & Index of UP, $k=0 . .7$ \\
\hline$n_{k}$ & Number of nodes of $\mathrm{UP}_{k}$ \\
\hline$R$ & Maximum retransmission limit \\
\hline$\sigma_{k}$ & $\begin{array}{l}\text { Probability that neither a } \mathrm{UP}_{k} \text { data frame nor its } \mathrm{ACK} \text { is } \\
\text { corrupted by noise }\end{array}$ \\
\hline$\lambda_{k}$ & $\begin{array}{l}\text { Data frame arrival probability during a CSMA slot for a } \\
\text { node of } \mathrm{UP}_{k}\end{array}$ \\
\hline$\tau_{k}$ & Access probability to medium by a $\mathrm{UP}_{k}$ node \\
\hline$g_{k}$ & $\begin{array}{l}\text { Probability that medium is idle during backoff countdown } \\
\text { for a UP } \mathrm{UP}_{k} \text { node }\end{array}$ \\
\hline$f_{k}$ & $\begin{array}{l}\text { Probability that medium remains idle in case of medium } \\
\text { access for a } \mathrm{UP}_{k} \text { node }\end{array}$ \\
\hline $\bar{L} L_{k, s}$ & $\begin{array}{l}\text { Mean successful data frame transmission time for a } \mathrm{UP}_{k} \\
\text { node in slots }\end{array}$ \\
\hline$L_{k, s o}$ & $\begin{array}{l}\text { Mean successful data frame transmission time of other } \\
\text { nodes for a given } \mathrm{UP}_{k} \text { node in slots }\end{array}$ \\
\hline$L_{k, c}$ & $\begin{array}{l}\text { Mean unsuccessful data frame transmission time for a } \\
\mathrm{UP}_{k} \text { node in slots }\end{array}$ \\
\hline$L_{k, c o}$ & $\begin{array}{l}\text { Mean unsuccessful data frame transmission time of other } \\
\text { nodes for a given } \mathrm{UP}_{k} \text { node in slots }\end{array}$ \\
\hline$\beta_{k}$ & $\begin{array}{l}\text { Probability that a data frame arrives to the queue during } \\
\text { the time interval between two successive Markov points }\end{array}$ \\
\hline$p_{k}$ & $\begin{array}{l}\text { Probability that indicates from the current CSMA slot to } \\
\text { the end of the RAP1 period for a UP } \mathrm{UP}_{k} \text { node there is not } \\
\text { enough time to complete a data frame transaction }\end{array}$ \\
\hline$\pi_{k, 0}$ & $\begin{array}{l}\text { Probability of an empty queue after serving a data frame } \\
\text { for a } \mathrm{UP}_{k} \text { node }\end{array}$ \\
\hline$p_{k, \text { Idle }}$ & $\begin{array}{l}\text { Probability of being in the idle state for a } \mathrm{UP}_{k} \text { node in } \\
\text { a CSMA slot }\end{array}$ \\
\hline$p_{s o, k}$ & $\begin{array}{l}\text { Probability of a successful transmission by the other } \\
\text { nodes for a given } \mathrm{UP}_{k} \text { node }\end{array}$ \\
\hline$p_{c o, k}$ & $\begin{array}{l}\text { Probability of an unsuccessful transmission by the other } \\
\text { nodes for a given } \mathrm{UP}_{k} \text { node }\end{array}$ \\
\hline$\Phi_{k}(z)$ & PGF for the duration of backoff process for a $\mathrm{UP}_{k}$ node \\
\hline $\mathcal{I}_{k}(z)$ & PGF of the idle period duration in slots \\
\hline $\mathcal{B}_{P, k, j}(z)$ & $\begin{array}{l}\text { PGF of the locked backoff counter because there is not } \\
\text { enough time to complete a frame transaction }\end{array}$ \\
\hline$\Theta_{k, j}(z)$ & $\begin{array}{l}\text { PGF of mean duration of locked backoff counter due } \\
\text { to a transmission on the medium, either successful or } \\
\text { unsuccessful }\end{array}$ \\
\hline$\zeta_{k}$ & Mean response time of a UP $k$ data frame \\
\hline
\end{tabular}

TABLE I

IMPORTANT PARAMETERS

assume that a $\mathrm{UP}_{k}$ node has a single $\mathrm{UP}_{k}$ queue. The network is considered to be single hop operating in $2.4 \mathrm{GHz}$ ISM band with uplink traffic only. The lengths of EAP1 and RAP1 are indicated by eap and rap in slots, respectively.

The control frames and headers are transmitted at $91.4 \mathrm{kbps}$ while the payload of the data frames is transmitted at 971.6 kbps. The size of the data frames for a node of $\mathrm{UP}_{k}$ is denoted by $l_{k}$ in slots and $l_{k, b}$ in bits and $a c k$ and $a c k_{b}$ indicate the sizes of an ACK frame in slots and in bits, respectively. We assume a Rician fading channel having BER as specified in eq. (1). Hence, data frame error rate is equal to $\sigma_{k}=(1-$ $B E R)^{l_{k, b}+a c k_{b}}$.

The analytical model is composed of three inter-related submodels; Markov chain, backoff duration, and queuing models.

\section{A. Markov Chain Model}

In the Markov chain model eight dependent 3-dimensional DTMCs (Discrete Time Markov Chains) for the eight UPs are introduced to formulate the medium access probabilities of the 
UPs.

Since the erroneous transmissions affect the mean numbers of considered CSMA slots in EAPs and RAPs in the DTMCs, the values of $X_{E}$ and $X_{R}$ are changed as follows:

$$
\begin{aligned}
X_{R} & =\frac{r a p-L_{7, s}}{f+\sum_{t=0}^{7} n_{t} \tau_{t} f_{t} \sigma_{t} L_{t, s}+\left(1-f-\sum_{t=0}^{7} n_{t} \tau_{t} f_{t} \sigma_{t}\right) L_{t, c}} \\
X_{E} & =\frac{e a p}{\chi+n_{7} \tau_{7} \psi \sigma_{7} L_{7, s}+\left(1-\chi-n_{7} \tau_{7} \psi \sigma_{7}\right) L_{7, c}}
\end{aligned}
$$

By solving the DTMCs we obtain 8 equations for $k=0 . .7$ as follows:

$$
1=p_{k, \text { Idle }}+Y_{k} \sum_{i=0}^{R}\left(1-f_{k} \sigma_{k}\right)^{i}\left(1+\sum_{j=1}^{W_{k, i}} \frac{W_{k, i}-j+1}{W_{k, i} g_{k, j}}\right)
$$

where $Y_{k}, k=0 . .7$, is the input probability to the zero-th backoff phase which is computed as follows:

$$
Y_{k}=\frac{\tau_{k} f_{k} \sigma_{k}\left(1-\pi_{k, 0}\right)+p_{k, \text { Idle }} \beta_{k}}{1-\left(1-f_{k} \sigma_{k}\right)^{R+1}\left(1-\pi_{k, 0}\right)}, \quad k=0 . .7
$$

$p_{k, \text { Idle }}$ is calculated as follows:

$$
p_{k, \text { Idle }}=\frac{\tau_{k} f_{k} \sigma_{k} \pi_{k, 0}^{\prime}}{\beta_{k}\left(1-\left(1-f_{k} \sigma_{k}\right)^{R+1}\right)}, \quad k=0 . .7
$$

Hence, the Markov chain model results in a set of eight equations while there are 16 unknown variables of $\tau_{k}, k=0 . .7$, and $\pi_{k, 0}, k=0 . .7$.

\section{B. Backoff Duration Model}

The average durations of every backoff phase and the total backoff period before successfully accessing the medium or dropping the data frame using PGFs are calculated in this section. In the extended model, the 4-dimensional DTMCs are remained unchanged compared to the presented model in [16].

$p_{s o, k}$ and $p_{c o, k}$ are computed as follows:

$$
\begin{aligned}
& p_{s o, k}=\sum_{i=0}^{7} \frac{n_{i} \tau_{i} f_{k} \sigma_{k}}{1-\tau_{i}}-\frac{\tau_{k} f_{k} \sigma_{k}}{1-\tau_{k}} \\
& p_{c o, k}=1-f_{k}-p_{s o, k}
\end{aligned}
$$

The PGF of the time interval between the moment when the backoff counter of a node of $\mathrm{UP}_{k}$ becomes equal to $j$ and the moment when the backoff counter becomes equal to $j-1$ at the $i$-th backoff phase is equal to:

$$
\Phi_{k, i, j}(z)=p_{k} \mathcal{B}_{P, k, j}(z)+\left(1-p_{k}\right)\left(f_{k} z+\Theta_{k, j}(z)\right)
$$

due to the fading channel the PGF of $i$-th backoff phase duration is computed as follows:

$$
\begin{aligned}
\Phi_{k, i}(z)= & \sum_{j=1}^{W_{k, i}} \prod_{t=1}^{j} \Phi_{k, i, t}(z)\left(f_{k} \sigma_{k}\left(L_{k, s} p_{k} z^{L_{k}}+1-L_{k, s} p_{k}\right)\right. \\
& \begin{array}{l}
(4,16),(4,8),(2,8),(1,4) \text { for } \mathrm{UP}_{0}, \mathrm{UP}_{1}, \mathrm{UP}_{2}, \mathrm{UP}_{3}, \mathrm{UP}_{4}, \mathrm{UP}_{5}, \\
\text { all the UPs. }
\end{array} \\
& \left.\left.+\left(1-f_{k} \sigma_{k}\right)\left(L_{k, c} p_{k} z^{L_{k}}+1-L_{k, c} p_{k}\right)\right) / W_{k, i}(9) \text { a he wively }\right\} . \text { The retry limit is set to } R=7 \text { for }
\end{aligned}
$$

The backoff process duration PGF for a $\mathrm{UP}_{k}$ node is given by

$$
\begin{aligned}
\Phi_{k}(z)= & \sum_{i=0}^{m_{k}}\left(\prod_{u=0}^{i} \Phi_{k, u}(z)\right)\left(1-f_{k} \sigma_{k}\right)^{i}\left(z^{L_{k, c}}\right)^{i} f_{k} \sigma_{k}+ \\
& \sum_{i=m_{k}+1}^{R}\left(\prod_{u=0}^{m_{k}} \Phi_{k, u}(z)\right) \Phi_{k, m_{k}}^{i-m_{k}}(z)\left(1-f_{k} \sigma_{k}\right)^{i} . \\
& \left(z^{L_{k, c}}\right)^{i} f_{k} \sigma_{k}+\left(\prod_{u=0}^{m_{k}} \Phi_{k, u}(z)\right) \Phi_{k, m_{k}}^{R-m_{k}}(z) . \\
& \left(1-f_{k} \sigma_{k}\right)^{R+1}\left(z^{L_{k, c}}\right)^{R+1}
\end{aligned}
$$

\section{Queuing Model}

The queueing model is developed to calculate accurate durations of idle periods and access probabilities of all UPs. The value of $\pi_{k, 0}$ is calculated as follows:

$$
\pi_{k, 0}=\frac{1-\rho_{k}}{\lambda_{k} E\left[\mathcal{I}_{k}(z)\right]}, k=0 . .7
$$

where $\rho_{k}=\lambda_{k} b_{k}$ is the traffic intensity of a $\mathrm{UP}_{k}$ node and $b_{k}=B_{k}^{\prime}(1)$ is the mean service time of a $\mathrm{UP}_{k}$ data frame. The PGF of service time (in slots) of a data frame for a $\mathrm{UP}_{k}$ node is computed as $B_{k}(z)=\Phi_{k}(z) \operatorname{St}_{k}(z)$.

The 16 unknown variables of $\tau_{k}$ and $\pi_{k, 0}$ for $k=0 . .7$ are calculated by the set of 16 equations of (4) and (11).

We are now able to calculate the mean response time of a $\mathrm{UP}_{k}$ data frame for an IEEE 802.15.6 CSMA-based WBAN in a fading channel. The frame response time is defined as the time interval from its arrival time to the time when it leaves the system, which is obtained as follows:

$$
\begin{aligned}
\zeta_{k}= & \frac{\lambda_{k} b_{k}^{(2)}-\lambda_{k} b_{k}}{2\left(1-\rho_{k}\right)}+\frac{E\left[\mathcal{I}_{k}(z)\left(\mathcal{I}_{k}(z)-1\right)\right]}{2 E\left[\mathcal{I}_{k}(z)\right]}+ \\
& \theta_{k}+\phi_{k}+\left(l_{k}+\text { sifs }+ \text { ack }\right)
\end{aligned}
$$

where $\theta_{k}=\frac{e a p^{2}}{2(e a p+r a p)}, k=0 . .6$ and $\theta_{7}=0$.

\section{Performance Evaluation}

In this section, we study the impacts of fading channel on MAC performance of an IEEE 802.15.6 CSMA-based WBAN under non-saturation regime. At first, we validate the analytical results by simulation results. Then, we investigate the performance of the network under different scenarios by presenting the simulation results only due to lack of space. We develop two performance measures of mean response time of data frames and successful transmission rate of the UPs. We use Maple 13 [17] for solving the analytical model while Opnet simulator [18] is used for simulation modeling. Analytical and simulation models closely follow assumptions and definitions from the IEEE 802.15.6 standard. We set the differentiation parameters of $\left(C W_{k, \min }, C W_{k, \max }\right)$ for all the UPs according to the standard $\{(16,64),(16,32),(8,32),(8,16)$, 


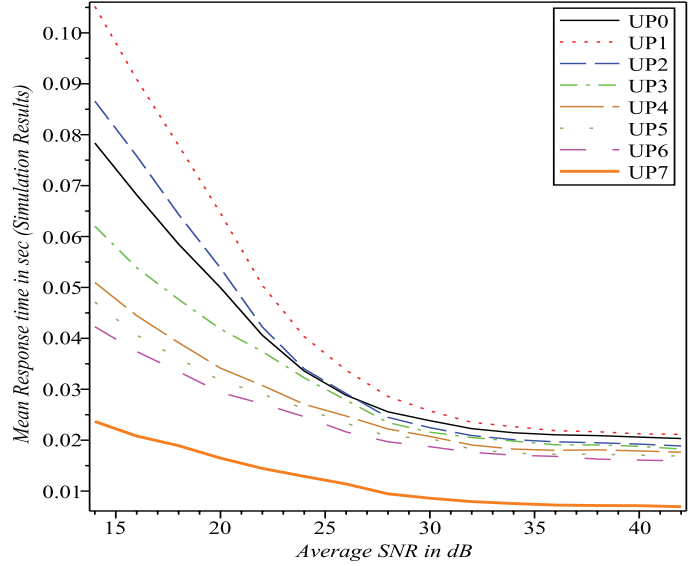

(a) $\mathrm{L}=1$

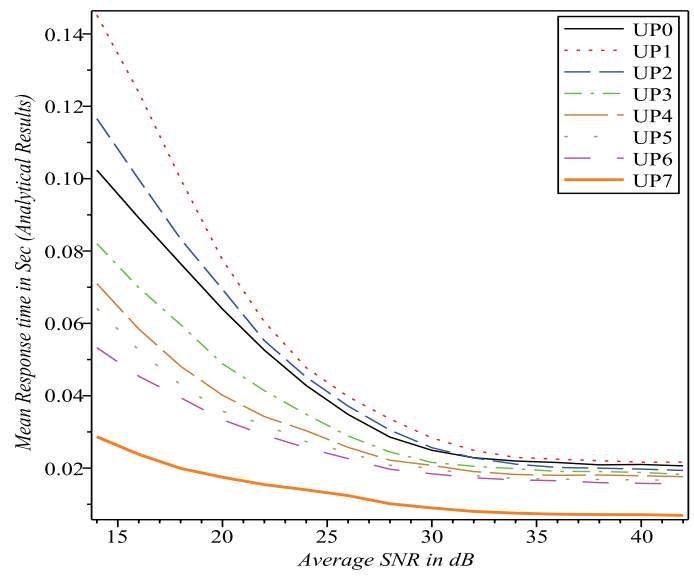

(b) $\mathrm{L}=1$

Fig. 2. Mean response time of data frames; $\left(K_{0}, K_{1}, K_{2}, K_{3}, K_{4}, K_{5}, K_{6}, K_{7}\right)=(4,1.5,3,3,2.5,1.5,1.5,4)$, $\left(\lambda_{0}, \lambda_{1}, \lambda_{2}, \lambda_{3}, \lambda_{4}, \lambda_{5}, \lambda_{6}, \lambda_{7}\right)=(2,2,20 / 3,2 / 3,1 / 3,2,2,2) \mathrm{p} / \mathrm{s}$

$n_{1}=2(\mathrm{ECG}-300 \mathrm{~B} / \mathrm{s}$ per node $), n_{2}=2(\mathrm{EMG}-1000 \mathrm{~B} / \mathrm{s}$ per node), $n_{3}=2$ (physical activity $-100 \mathrm{~B} / \mathrm{s}$ per node), $n_{4}=4$ (glucose, oxygen saturation, temperature, respiratory - 50B/s per node), $n_{5}=2$ (ECG, blood pressure $-300 \mathrm{~B} / \mathrm{s}$ per node), $n_{6}=2(\mathrm{EEG}-300 \mathrm{~B} / \mathrm{s}$ per node $), n_{7}=2(\mathrm{ECG}-300 \mathrm{~B} / \mathrm{s}$ per node). We follow two different scenarios for investigating the error performance of the network. In the first scenario, we assume that the data frame sizes of all UPs are equal to $150 \mathrm{~B}$. Through this scenario we evaluate the impacts of different Rician factors of channels between nodes and the hub on performance of the network by varying the SNR values. In the second scenario different UPs may have different channel qualities (different Rician factors) and different frame sizes. The data frame sizes of different UPs are set based on the data rate and the data frame delay sensitivity of the UPs.

In all plots in this section, the lines with the line-styles thin solid (black), dot (red), dash (blue), dash-dot (green), long-dash (gold), space-dot (khaki), space-dash (magenta), and thick solid (coral) represent user priorities, 0, 1, 2, 3, 4,

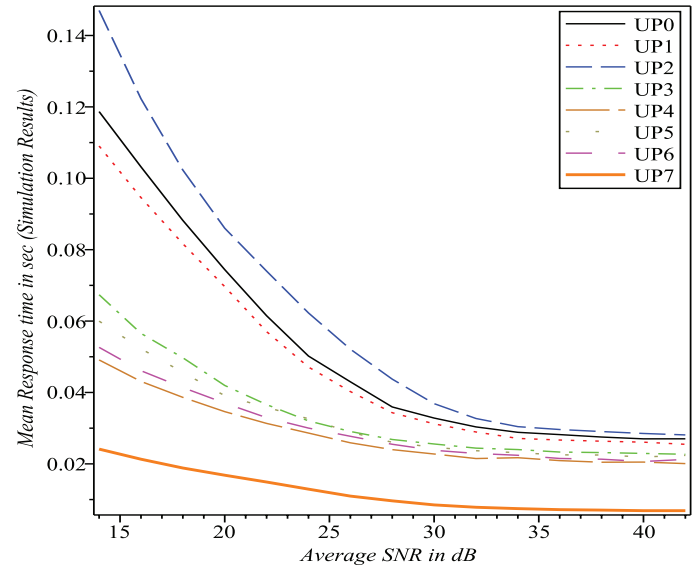

(a) $\mathrm{L}=1$

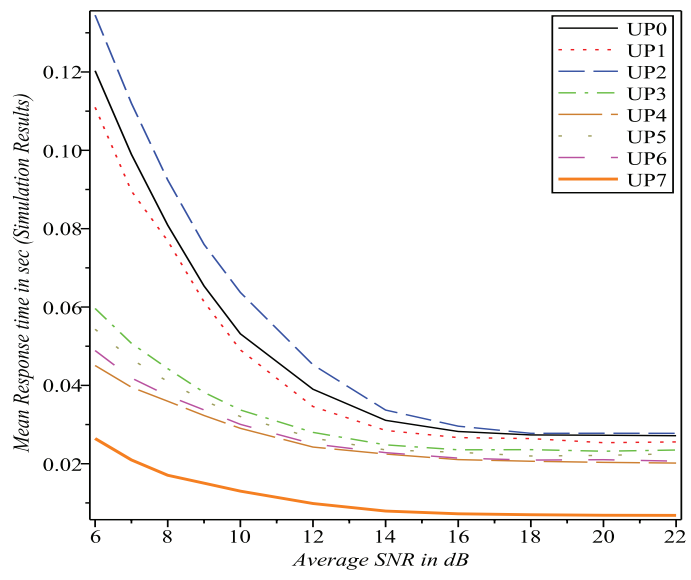

(b) $\mathrm{L}=2$

Fig. 3. Mean response time of data frames; rap $=0.3 \mathrm{~s}$ eap $=0.1 \mathrm{~s}$, different frame sizes $\left(l_{0}, l_{1}, l_{2}, l_{3}, l_{4}, l_{5}, l_{6}, l_{7}\right)=(300,150,500,50,50,150,150,150) \mathrm{B}$, $\left(K_{0}, K_{1}, K_{2}, K_{3}, K_{4}, K_{5}, K_{6}, K_{7}\right)=(4,1.5,3,3,2.5,1.5,1.5,4)$, $\left(\lambda_{0}, \lambda_{1}, \lambda_{2}, \lambda_{3}, \lambda_{4}, \lambda_{5}, \lambda_{6}, \lambda_{7}\right)=(1,2,2,2,1,2,2,2) \mathrm{p} / \mathrm{s}$

\section{5,6 , and 7 , respectively.}

The mean response times of the UPs for the first scenario are depicted in Fig. 2. The Rician factors for the channels between the hub and the nodes of $\mathrm{UP}_{k}, k=0 . .7$ are set to $4,1.5,3$, $3,2.5,1.5,1.5$, and 4 , respectively. The arrivals rates of the nodes are set to $2,2,20 / 3,2 / 3,1 / 3,2,2$, and 2 packets/sec for $\mathrm{UP}_{k}, k=0 . .7$, respectively. In addition, the lengths of RAP1 and EAP1 are set to rap $1=0.5 s$ and eap $1=0.1 s$. Fig. 2(a) shows the simulation results when the diversity order is set to 1 while Fig. 2(b) depicts the analytical results in case of diversity order of 1 . The results indicate that the channel quality strongly affects the response time of data frames since though the $\mathrm{UP}_{1}$ nodes have higher priority than $\mathrm{UP}_{0}$ nodes the data frames of former nodes obtain longer response time. As Fig. 2 indicates the simulation and the analytical results acceptably match.

In the second scenario, the data frame sizes of different UPs are set to $300 \mathrm{~B}, 150 \mathrm{~B}, 500 \mathrm{~B}, 50 \mathrm{~B}, 50 \mathrm{~B}, 150 \mathrm{~B}, 150 \mathrm{~B}$, and $150 \mathrm{~B}$ for $\mathrm{UP} k, k=0 . .7$. We study the error performance 


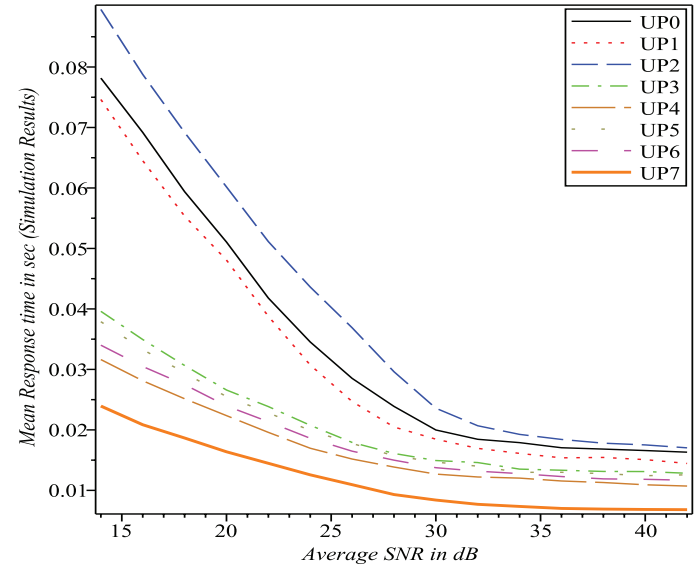

(a) $\mathrm{L}=1$

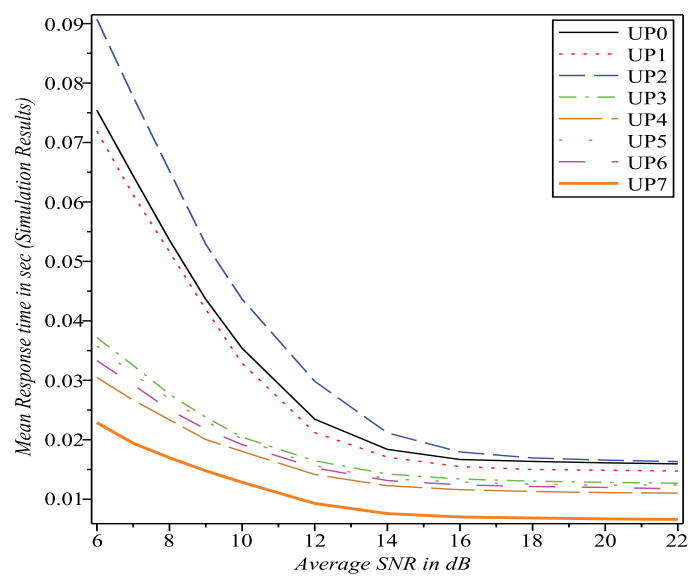

(b) $\mathrm{L}=2$

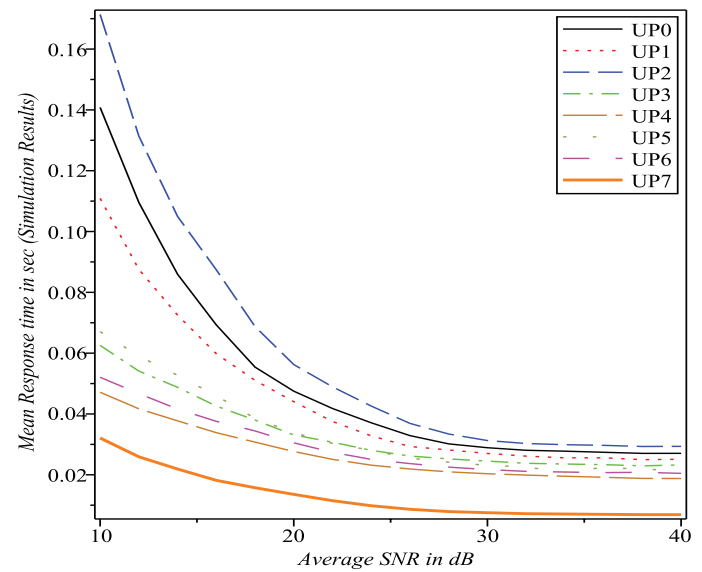

(a) $\mathrm{L}=1$

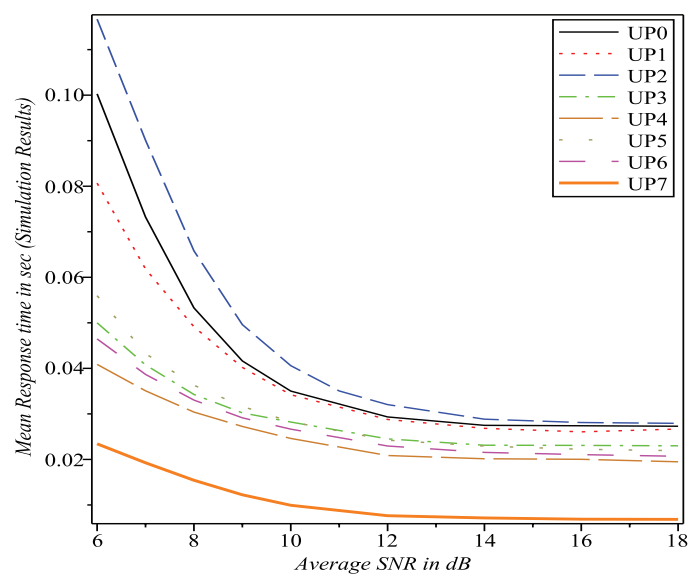

(b) $\mathrm{L}=2$
Fig. 4. Mean response time of data frames; rap $=1 \mathrm{~s}$ eap $=0.1 \mathrm{~s}$, different frame sizes $\quad\left(l_{0}, l_{1}, l_{2}, l_{3}, l_{4}, l_{5}, l_{6}, l_{7}\right)=(300,150,500,50,50,150,150,150) \mathrm{B}$, $\left(K_{0}, K_{1}, K_{2}, K_{3}, K_{4}, K_{5}, K_{6}, K_{7}\right)=(4,1.5,3,3,2.5,1.5,1.5,4)$, $\left(\lambda_{0}, \lambda_{1}, \lambda_{2}, \lambda_{3}, \lambda_{4}, \lambda_{5}, \lambda_{6}, \lambda_{7}\right)=(1,2,2,2,1,2,2,2) \mathrm{p} / \mathrm{s}$

of the WBAN through 3 different experiments. In the first two experiments the Rician factors are set as $K_{0}=1.5, K_{1}=$ $4, K_{2}=3, K_{3}=3, K_{4}=2.5, K_{5}=1.5, K_{6}=1.5, K_{7}=4$. The only difference between the first and the second experiments is the lengths of RAP1, which are respectively set to $r a p=0.3 s$ and $r a p=1 s$ while the EAP1 length is equal to eap $=0.1 s$. The arrival rates of the nodes for both experiments are set to $\lambda_{0}=1 p / s, \lambda_{1}=2 p / s, \lambda_{2}=2 p / s, \lambda_{3}=2 p / s, \lambda_{4}=$ $1 p / s, \lambda_{5}=2 p / s, \lambda_{6}=2 p / s, \lambda_{7}=2 p / s$. The results for the first experiment are shown in Fig. 3 for the cases where the diversity order is set to $\mathrm{L}=1$ and $\mathrm{L}=2$. Fig. 6 depicts the mean response times of data frames for all UPs for the second experiment where the diversity order is equal to 1 and 2 . Comparing the results of the experiments show that increasing the ratio of RAP1 length and EAP1 length generally improves the performance of all UPs in the WBAN since the channel is more efficiently utilized. The third experiment belongs to the case where the Rician factors are changed compared to the all other experiments in this work. We set the Rician
Fig. 5. Mean response time of data frames; rap $=0.3 \mathrm{~s}$ eap $=0.1 \mathrm{~s}$, different frame sizes $\quad\left(l_{0}, l_{1}, l_{2}, l_{3}, l_{4}, l_{5}, l_{6}, l_{7}\right)=(300,150,500,50,50,150,150,150) \mathrm{B}$ $\left(K_{0}, K_{1}, K_{2}, K_{3}, K_{4}, K_{5}, K_{6}, K_{7}\right)=(2.2,4.9,4.9,4.9,4.9,4.9,2.2,4.9)$, $\left(\lambda_{0}, \lambda_{1}, \lambda_{2}, \lambda_{3}, \lambda_{4}, \lambda_{5}, \lambda_{6}, \lambda_{7}\right)=(1,2,2,2,1,2,2,2) \mathrm{p} / \mathrm{s}$

factors in this experiment as $K_{0}=2.2, K_{1}=4.9, K_{2}=$ $4.9, K_{3}=4.9, K_{4}=4.9, K_{5}=4.9, K_{6}=2.2, K_{7}=4.9$. The data frame sizes are set to the same values of the first two experiments in this scenario. Arrival rates of this experiment are also kept unchanged. The length of RAP1 and EAP1 are set to $r a p=0.3 s$ and eap $=0.1 s$, respectively. The main finding of the second scenario (all three experiments) is that the data frame size is the most effective parameter on error performance of an IEEE 802.15.6 CSMA-based WBAN. In all three experiments the $\mathrm{UP}_{2}$ nodes have the largest data frame response times since they have the largest frame size (500B).

Fig. 6 depicts the successful transmission rate of all UPs in the WBAN where $r a p=1 s$, eap $=0.1 s, K_{0}=1.5, K_{1}=$ $4, K_{2}=3, K_{3}=3, K_{4}=2.5, K_{5}=1.5, K_{6}=1.5, K_{7}=4$, and $\lambda_{0}=1 p / s, \lambda_{1}=2 p / s, \lambda_{2}=2 p / s, \lambda_{3}=2 p / s, \lambda_{4}=$ $1 p / s, \lambda_{5}=2 p / s, \lambda_{6}=2 p / s, \lambda_{7}=2 p / s$. The results indicate that the data frame size causes the largest successful transmission rate degradation. However, under non-saturation condition, the Channel Rician factor is not as effective as the 


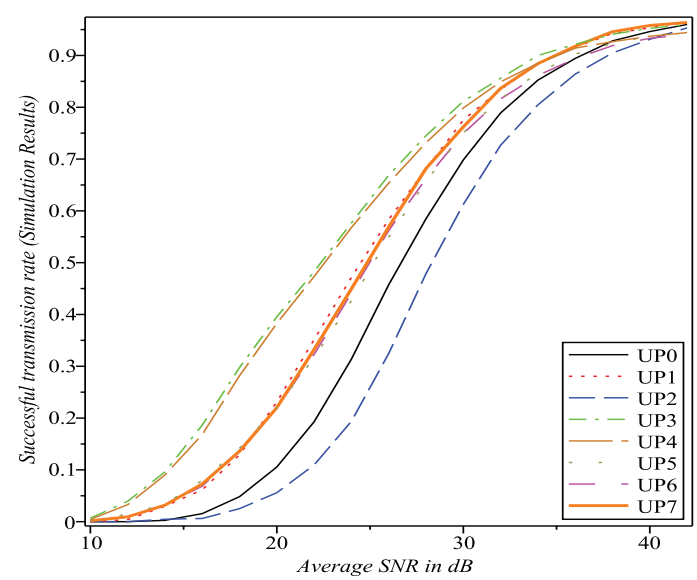

(a) $\mathrm{L}=1$

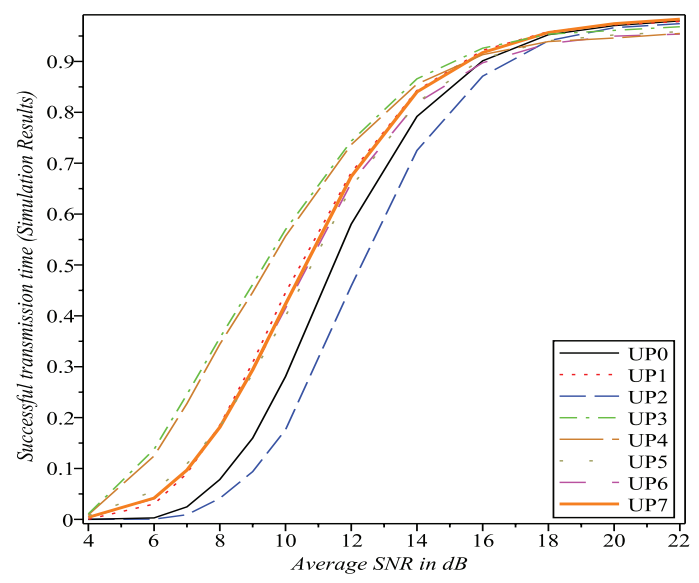

(b) $\mathrm{L}=2$

Fig. 6. Successful transmission rate; rap $=1 \mathrm{~s}$ eap $=0.1 \mathrm{~s}$, different frame sizes $\quad\left(l_{0}, l_{1}, l_{2}, l_{3}, l_{4}, l_{5}, l_{6}, l_{7}\right)=(300,150,500,50,50,150,150,150) \mathrm{B}$, $\left(K_{0}, K_{1}, K_{2}, K_{3}, K_{4}, K_{5}, K_{6}, K_{7}\right)=(4,1.5,3,3,2.5,1.5,1.5,4)$, $\left(\lambda_{0}, \lambda_{1}, \lambda_{2}, \lambda_{3}, \lambda_{4}, \lambda_{5}, \lambda_{6}, \lambda_{7}\right)=(1,2,2,2,1,2,2,2) \mathrm{p} / \mathrm{s}$

data frame size on successful transmission rate of the UPs. Though the Rician factors for UPs of 1, 3, 6, and 7 are different $(4,3,1.5$, and 4 , respectively) they almost achieve equal successful transmission rates. The $\mathrm{UP}_{2}$ and $\mathrm{UP}_{0}$ nodes obtain the lowest successful transmission rates due to their large data frame sizes.

According to the results of this section, we can set the transmission powers of the nodes having different UPs to the lowest values to fulfil the maximum error tolerance of the nodes. The transmission powers should be chosen based on the channel quality and data frame sizes, as the most effective parameters on the error performance of the WBANs. Appropriate transmission powers and study of the error performance of WBAN are important and necessary since different nodes have different performance due to their transmission medium quality, which is caused by their location on or in the body.

\section{CONCLUSION}

In this paper, we studied the impacts of channel quality, data frame size, diversity order, data frame arrival rate, access phase lengths, and UPs on error performance of an IEEE 802.15.6 CSMA-based WBAN. We showed how varying SNR values for different scenarios affects the MAC performance descriptors of the network. The results indicate that increasing the diversity order and transmission power generally improves the MAC performance as well. We found that the data frame sizes and the channel quality between nods and the hub are the most effective parameters on the WBAN error performance.

\section{REFERENCES}

[1] Wireless Body Area Network Draft Standard, IEEE Std. 802.15.6, 2010.

[2] A. F. Molisch, Wireless Communications. John Wiley and Sons, 2010.

[3] A. Taparugssanagorn, A. Rabbachin, M. Hmlinen, J. Saloranta, and J. Iinatti, "A review of channel modelling for wireless body area network in wireless medical communications," in Proc. IEEE WPMC, Lapland, Finland, 2008.

[4] S. L. Cotton and W. G. Scanlon, "An experimental investigation into the influence of user state and environment on fading characteristics in wireless body area networks at $2.45 \mathrm{GHz}$," IEEE Transactions on Wireless Communications, vol. 8, pp. 6-12, 2009.

[5] Y. I. Nechayev, P. S. Hall, and Z. H. Hu, "Characterisation of narrowband communication channels on the human body at $2.45 \mathrm{GHz}$," IEEE Microwaves, Antennas \& propagation, vol. 4, pp. 722-732, 2010.

[6] I. Khan, Y. I. Nechayev, and P. S. Hall, "On-body diversity channel characterization," IEEE Transactions on Antennas and propagation, vol. 58, pp. 573-580, 2010.

[7] Z. H. Hu, Y. I. Nechayev, P. S. Hall, C. C. Constantinou, and H. Yang, "Measurements and statistical analysis of on-body channel fading at 2.45 GHz," IEEE Antennas and Wireless Propagation Letters, vol. 6, pp. 612-615, 2007.

[8] S. L. Cotton and W. G. Scanlon, "Measurements and statistical analysis of on-body channel fading at $2.45 \mathrm{GHz}$," IEEE Antennas Wireless Propagation Letters, vol. 6, pp. 51-55, 2007.

[9] — "A statistical analysis of indoor multipath fading for a narrowband wireless body area network," in Proc. IEEE PIMRC, Helsinki, Finland, 2006, pp. $1-5$.

[10] T. T. Tjhung, C. Loo, and N. P. Secord, "BER performance of DQPSK in slow rician fading," IEEE Electronics Letters, vol. 28, pp. 1763-1765, 1992.

[11] C. Tellambura and V. K. Bhargava, "Unified error analysis of DQPSK in fading channels," IEEE Electronics Letters, vol. 30, pp. 2110-2111, 1994.

[12] M. K. Simon and M. S. Alouini, Digital communications over fading channel. John Wiley, 2005.

[13] J. Sun and I. S. Reed, "Linear diversity analysis for M-PSK in rician fading channels," IEEE Transactions on Communications, vol. 51, pp. 1749-1753, 2003.

[14] J. G. Proakis and M. Salehi, Digital Communications (5th Ed.). McGraw-Hill, 2008.

[15] M. G. Shayesteh, "Exact symbol and bit error probabilities of linearly modulated signals with maximum ratio combining diversity in frequency nonselective rician and rayleigh fading channels," IET Communications, vol. 5, pp. 12-26, 2011.

[16] S. Rashwand and J. Misic, "Performance evaluation of IEEE 802.15.6 under non-saturation condition," in Proc. the IEEE Global Telecommunications Conference (Globecom11), Houston, Texas, US., Dec. 2011.

[17] (2010) Maple 13, maplesoft. Waterloo, Canada. [Online]. Available: http://www.maplesoft.com

[18] (2010) Opnet modeler, opnet technologies, inc. Bethesda, MD. [Online]. Available: http://www.opnet.com/ 